\title{
Pathobiochemie und klinisch-chemische Diagnostik der hepatogenen Enzephalopathie und des Coma hepaticum
}

\author{
A. M. Gressner \\ Abteilung für Klinische Chemie und Zentrallaboratorium, \\ Klinikum der Philipps-Universität, Marburg
}

\begin{abstract}
Zusammenfassung:
Hepatogene Enzephalopathie und Coma hepaticum beruhen auf einer funktionell-metabolischen Schädigung des Hirns infolge schwerer Leberzellinsuffizienz und/oder portosystemischer Umgehung der Leber. Sie sind verbunden mit einer Akkumulation cerebrotoxischer Substanzen im Blut, Urin und Liquor (Ammonium, Mercaptane, kurzkettige Fettsäuren, Phenol und Phenolderivate) sowie mit schweren Veränderungen im Aminosäurespektrum gekennzeichnet durch eine Zunahme aromatischer (Tyrosin, Phenylalanin, Tryptophan) und Abnahme verzweigtkettiger (Leucin, Isoleucin, Valin) Aminosäuren. Die komplexen biochemischen Anomalien lassen sich pathogenetisch einordnen in die Intoxikationshypothese, der zufolge cerebrotoxische Substanzen für die neuropsychiatrischen Veränderungen verantwortlich sind und in die Neurotransmitterhypothese, die von der intracerebralen Bildung inaktiver (z. B. Octopamin, $\beta$-Phenylethanolamin) oder inhibitorischer (z. B. Serotonin, GABA) Neurotransmitter bei gehemmter Synthese exzitatorischer Transmitter (Dopamin und Noradrenalin) ausgeht. Sowohl die Bestimmung hirntoxischer Metabolite als auch die Erfassung von falschen oder inaktiven Neurotransmittern (besonders von Octopamin) im Serum, gegebenenfalls auch im Urin oder Liquor sind für die Diagnostik, Verlaufskontrolle und prognostische Beurteilung des Coma hepaticums verwertbar.
\end{abstract}

Schlüsselwörter:

Coma hepaticum - hepatogene Enzephalopathie - cerebrotoxische Metabolite - falsche Neurotransmitter

\section{Summary:}

Hepatic encephalopathy and coma hepaticum are caused by a metabolic injury of the brain due to severe liver failure and/or portosystemic shunt. They are associated with an accumulation of cerebrotoxic metabolites in blood, urine, and cerebrospinal fluid (ammonia, mercaptans, short-chain fatty acids, phenols) and changes of the amino acid profile characterized by an increase of aromatic (tyrosine, phenylalanine, tryptophan) and decrease of branched-chain (leucine, isoleucine, valine) amino acids. The complex biochemical abnormalities can be classified in an "intoxication hypothesis", which is based upon the action of cerebrotoxic compounds and in the "neurotransmitter hypothesis", which is based upon the cerebral synthesis of inactive (e.g. octopamine, $\beta$-penylethanolamine) or inhibitory (e.g. serotonin, GABA) neurotransmitter in combination with the decreased synthesis of physiologic transmitters dopamine and noradrenaline. Both the estimation of cerebrotoxic metabolites and the measurement of false neurotransmitters (especially of octopamine) in serum or even in urine and cerebrospinal fluid can be utilized for diagnosis, follow-up and prognosis of coma hepaticum.

\section{Keywords:}

Coma hepaticum - hepatic encephalopathy - cerebrotoxic metabolites - false neurotransmitter

Die hepatische Enzephalopathie ist eine nicht-entzündliche, funktionell-metabolische Schädigung des Gehirns auf der Basis einer akuten oder chronischen massiven Leberzellinsuffizienz. Sie äußert sich in einer komplexen neuropsychiatrischen Symptomatik, die sich von Konzentrations- und Merkschwäche, Reizbarkeit, Antriebsschwäche über Verwirrtheit und delirante Zustände, Flattertremor („flapping tremor"), Schläfrigkeit und weiteren neuromuskulären Symptomen bis hin zum Stupor und Coma, dem finalen und in vielen Fällen letalen Stadium der Enzephalopathie erstreckt. Die verschiedenen Schweregrade der hepatogenen Enzephalopathie werden im allgemeinen in 4 Stadien eingeteilt (1): Stadium I (Prodromalstadium), Stadium II (drohendes Coma), Sta- dium III (Stupor) und Stadium IV (tiefes Coma). Nach Art der zugrundeliegenden Lebererkrankung wird zwischen dem endogenen Leberzerfallscoma und dem Leberausfallscoma unterschieden (2). Das relativ seltene, nur für etwa $10 \%$ aller Comafälle verantwortliche Leberzerfallscoma (3) kann bei einer fulminant verlaufenden ausgeprägten Leberschädigung ohne vorherige Lebererkrankung aufgrund toxischer (Phalloidin, Paracetamol, Halothan, Tetrachlorkohlenstoff u.a.), infektiöser (fulminant verlaufende Virushepatitis, Rickettsieninfektion) oder zirkulatorischer (akute Lebervenenthrombose) Ursachen akut eintreten. Häufiger, mit ca. $60 \%$ der Fälle ist das exogene Leberausfallscoma Ursache einer schweren hepatogenen Enzephalopathie (3). Es entwickelt sich im 
allgemeinen protrahiert, oft mit akuten Exazerbationen verbunden über mehrere Stadien hinweg zum Finalzustand und tritt am häufigsten als Folge einer alkohol-toxischen oder posthepatitischen Leberzirrhose auf. In Gegensatz zum endogenen Zerfallscoma ist das Ausfallscoma mit einem oft ausgeprägten portosystemischen Umgehungskreislauf der Leber verbunden (portosystemische Enzephalopathie). In ca. 30\% der Comata liegen Mischformen von Zerfalls- und Ausfallscoma vor, z.B. bedingt durch Fettleber-Coma, akute nektrotische Schübe bei Leberzirrhose oder durch eine die Zirrhose überlagernde Serumhepatitis (3).

Obwohl die Pathomechanismen der akuten und chronischen hepatitischen Enzephalopathie bzw. des Comas im einzelnen wahrscheinlich unterschiedlich und multifaktorieller Genese sind, lassen sich folgende gemeinsame pathobiochemische Grundzüge herausstellen (4-8).

a) Akkumulation zerebro-toxischer Substanzen in der systemischen Zirkulation infolge einer stark reduzierten hepatischen Clearance durch Parenchymschiwund und/ oder portosystemischer Umgehung der Leber,

b) der überwiegende Teil der akkumulierenden toxischen Substanzen entsteht im Darm und ist Proteinursprungs,

c) die Enzephalopathie kann folglich (siehe b) durch orale Proteinbelastung oder Blutungen in das Intestinallumen akut verstärkt oder ausgelöst bzw. durch Restriktion der oralen Proteinzufuhr oder antibiotischen Hemmung des mikrobiellen Proteinabbaues vermindert werden,

d) die Beeinträchtigung der neurosynaptischen Überleitung durch Veränderungen der neuronalen Membranfunktion, der Neurotransmitterzusammensetzung und/oder des zerebralen Energiestoffwechsels (reduzierter $\mathrm{O}_{2}$ - und Glukoseverbrauch).

Sowohl bei Patienten mit hepatogener Enzephalopathie als auch bei tierexperimenteller portocavaler Shuntbildung sind erhöhte Konzentrationen von Metaboliten nachgewiesen worden, die häufig im Synergismus mit

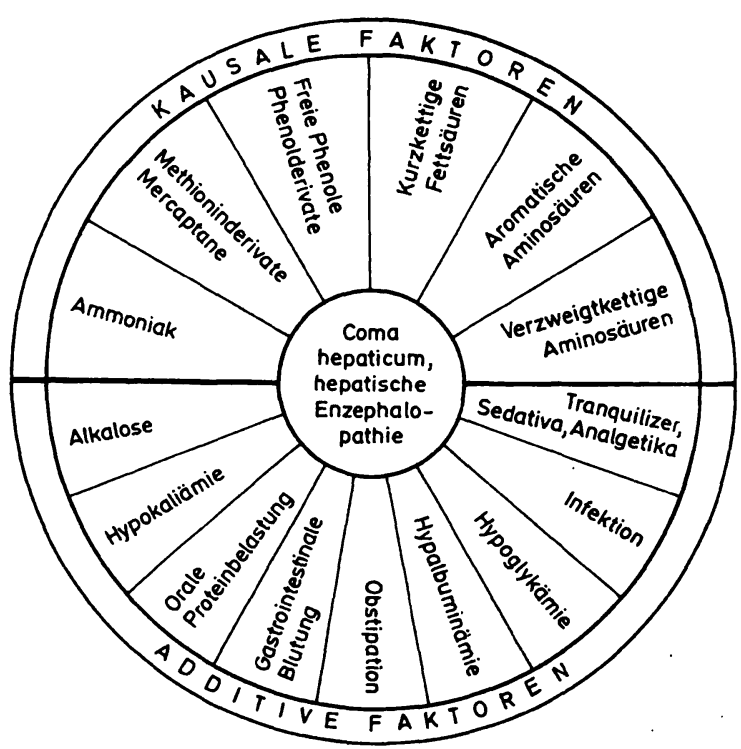

Abb. 1: Ursächliche und erschwerende Faktoren des Coma hepaticums und der hepatischen Enzephalopathie. Erklärung im Text

Lab.med. 9: 232 (1985) anderen Faktoren an der Pathogenese des Coma hepaticum direkt oder indirekt beteiligt sind, wenngleich ihr Wirkungsmechanismus im einzelnen oft noch nicht geklärt ist (Abb. 1) $(5,8)$. Die wichtigsten Metabolitverschiebungen betreffen:

- erhöhte Konzentrationen von Ammoniak in Blut, Muskel, Gehirn und Liquor,

- erhöhte Konzentrationen von Glutamin und $\%$-Ketoglutaramid in Gehirn, Liquor und Muskel,

- erhöhte Konzentrationen kurzkettiger Fettsäuren in Blut und Liquor,

- Auftreten von Mercaptanen in Blut, Liquor, Gehirn, Urin und Atemluft („Foetor hepaticus”),

- Auftreten von unkonjugierten (freien) Phenolen und Phenolderivaten in Blut und Urin,

- Verschiebungen des Aminosäureprofils in Blut, Gehirn, Liquor und Urin, gekennzeichnet durch Zunahme der aromatischen Aminosäuren Tryptophan (nicht-proteingebundener Anteil), Phenylalanin, Tyrosin, sowie Aspartat und Glutamat und des Methionins bei gleichzeitiger Abnahme der verzweigtkettigen Aminosäuren Leucin, Isoleucin und Valin. Weiterhin Zunahme der Tyrosinmetabolite Tyramin und Phenole,

- erhöhte Konzentrationen falscher Neurotransmitter wie Octopamin und $\beta$-Phenylethanolamin in Blut, Gehirn, Liquor, Urin und Muskel,

- erniedrigte Konzentrationen echter Neurotransmitter (Dopamin, Noradrenalin) in Gehirn und Muskel.

Die komplexen Stoffwechselveränderungen lassen sich hinsichtlich ihrer Beteiligung an der Pathogenese des Coma hepaticum in die beiden wesentlichen Hypothesen, die zur Erklärung der Enzephalopathie herangezogen werden, einordnen:

- Intoxikationshypothese

- Neurotransmitterhypothese.

\section{Intoxikationshypothese}

Dieser Hypothese liegt die experimentell gut begründete Annahme zugrunde, daß durch die eingeschränkte Entgiftungsfunktion der Leber Substanzen auftreten, die nach Übertritt in den Liquorraum toxische Wirkungen auf den Hirnstoffwechsel ausüben. Die wichtigsten dieser Toxine sollen im folgenden.kurz berschrieben werden.

\section{Ammoniak}

Die Leber nimmt durch ihre Fähigkeit zur Harnstoffsynthese als Ort der definitiven Elimination des Ammoniums (definiert als die Summe des freien $\mathrm{NH}_{3}$ und des mit ihm im $\mathrm{pH}$-abhängigen Dissoziationsgleichgewicht stehenden $\mathrm{NH}_{4}^{+}$

$$
\mathrm{NH}_{4}^{+} \underset{<\mathrm{pH}}{\stackrel{>\mathrm{pH}}{\rightleftharpoons}} \mathrm{NH}_{3}+\cdot \mathrm{H}^{+}
$$

das bei normalem Blut-pH 7,4 zu 98\% auf der Seite von $\mathrm{NH}_{4}^{+}$liegt) eine zentrale Stellung in der Beseitigung des toxisch wirkenden Ammoniums ein. Das überwiegend (aber nicht ausschließlich) im Darmlumen bakteriell gebildete und absorbierte Ammonium (ca. $4 \mathrm{~g} / \mathrm{Tag}$ ) gelangt in erhöhtem Maße in die systemische Zirkulation aufgrund der portosystemischen Umgehung der Leber sowie 
ihrer stark herabgesetzten Fähigkeit zur Harnstoffsynthese. Letztere läßt sich an der signifikanten Aktivitätsabnahme der Schrittmacherenzyme des Harnstoffzyklus, der Carbamylphosphatsynthetase und der ArgininosuccinatSynthetase, in der zirrhotischen Leber nachweisen (9). Die daraus resultierende Hyperammoniämie, deren Ausmaß nur bedingt mit dem Schweregrad der Enzephalopathie korreliert $(4,7,10,11)$ und bei etwa $10 \%$ der Patienten mit Coma hepaticum nicht angetroffen wird (7), führt zu einem gesteigerten Influx von Ammonium in den Liquorraum und cerebralen Aufnahme (bevorzugt graue Substanz). Hier wird es durch Gliazellen mit Glutamat zum Glutamin umgesetzt (Abb. 2), dessen Konzentration im Hirn und Liquor stark ansteigt. Der Glutaminspiegel im Liquor korreliert von allen zur Verfügung stehenden biochemischen Parametern am besten mit dem Schweregrad der hepatischen Enzephalopathie $(4,5)$. Daneben entsteht aus der Bindung von Ammonium an $\alpha-$ Ketoglutarat in höheren Konzentrationen $\alpha$-Ketoglutaramid, welches, wie Injektionsversuche in die Vertikel von Tieren gezeigt haben, zumindest für einen Teil der zerebrotoxischen Wirkung des Ammoniums verantwortlich zu machen ist. Ein weiterer Teil der toxischen Hirnwirkungen ist möglicherweise dem Glutamin zuzuschreiben, welches in Kompetition mit Glutamat in die peripheren Nervenendigungen aufgenommen wird und dort als inaktiver
Neutrotransmitter anstelle der exzitatorischen Aminosäure Glutamat fungiert (12). Eine dem Ammonium zugeschriebene Hemmung des zerebralen Energiestoffwechsels sowie direkte Effekte auf die neuronalen Membranen kommen als weitere pathobiochemische Mechanismen seiner Hirntoxizität in Betracht. Eine in allen Punkten befriedigende, experimentell bewiesene Vorstellung über die Ammoniumwirkung auf den Hirnstoffwechsel gibt es zur Zeit noch nicht. Es ist anzunehmen, daß einige der Ammoniakwirkungen synergistisch durch andere Toxine, z. B. kurzkettige Fettsäuren und Mercaptane (s.u.), verstärkt werden. Auf die Auswirkungen einer durch Hyperammoniämie bewirkten gesteigerten Glukagonsekretion wird unten eingegangen.

Ausmaß des Anstieges und Toxizität des Ammoniums werden durch einige additive Faktoren beeinflußt (Abb. 1), die z.T. für die nur mangelhafte Korrelation der Hyperammoniämie zum klinischen Schweregrad des $\mathrm{Co}$ mas verantwortlich sind $(3,7,10,11)$.

\section{a) Alkalose}

Mit zunehmendem $\mathrm{pH}$ verschiebt sich das Äquilibrium zwischen Ammoniumionen $\left(\mathrm{NH}_{4}^{+}\right)$und freiem Ammoniak $\left(\mathrm{NH}_{3}\right)$, welches bei $\mathrm{pH} 7,4$ auf der Seite des $\mathrm{NH}_{4}^{+}$liegt (s.o.) stark zugunsten der nicht-ionisierten Form. Nur die

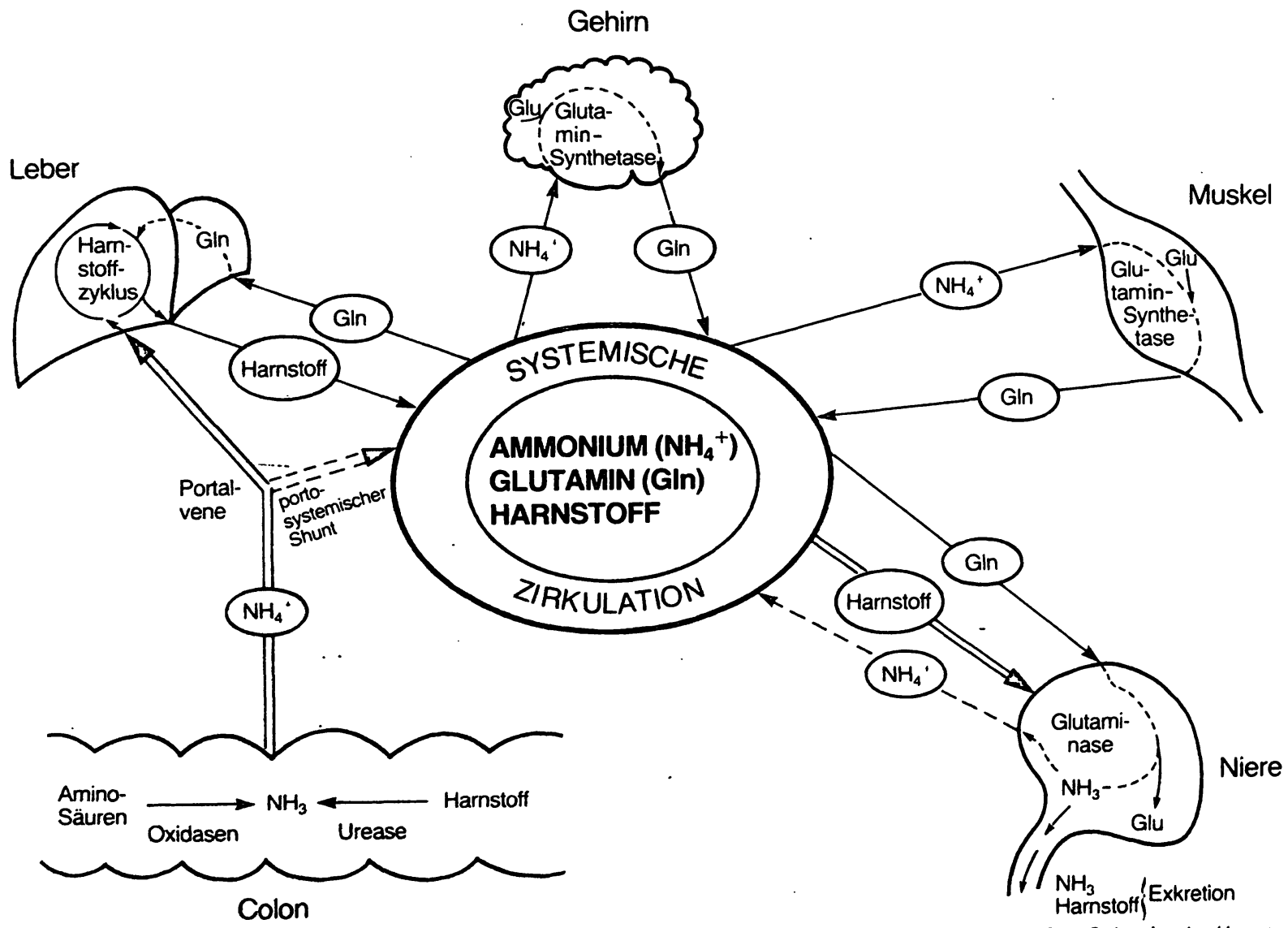

Abb. 2: Zusammenstellung der am Ammoniumstoffwechsel beteiligten Organe (modifiziert nach Rel. 48). Das Colon ist der Hauptbildungsort des $\mathrm{NH}_{4}^{+}$, wo es durch Wirkung bakterieller Urease und Aminosäureoxidasen entsteht. In der Leber erfolgt die Umwandlung

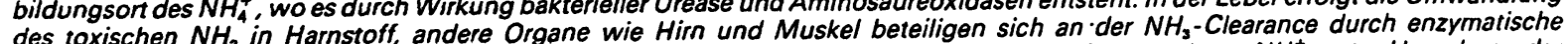

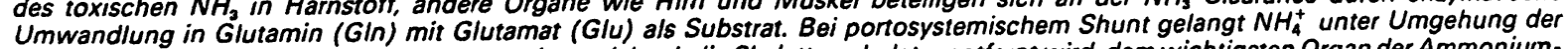
Leber direkt in die Zirkulation, aus der es vorwiegend durch die Skelettmuskulatur entfernt wird, dem wichtigsten Organ der AmmoniumLeber direkt in die Zirkulation, aus der es vorwiegend durch die Skelettmuskulatur entfernt wird, dem wichtigster arteriellen Ammoniumkonzentration 
letztere, nicht jedoch Ammoniumionen kann Zellmembranen und die Blut-Hirn-Schranke permeieren und somit intrazelluläre Wirkungen entfalten.

\section{b) Hypokaliämie}

Häufig im Rahmen eines sekundären Hyperaldosteronismus bei Leberzirrhose oder durch Diuretika induziert, erhöht durch Aktivitätssteigerung der renalen Glutaminase die Ammoniumkonzentration im zurückströmenden Nierenvenenblut.

c) Gastrointestinale Blutungen (z.B. Osophagusvarizen), orale Proteinbelastung und Obstipation führen zu vermehrtem Anfall von Substraten mikrobieller Ammoniumbildung und Freisetzung aromatischer Aminosäuren (s.u.) bzw. bewirken eine gesteigerte enterale Absorption von Ammonium.

d) Katabole Bedingungen (z. B. im Rahmen von Infektionen) führen zu einer vermehrten Bildung von Ammonium unter Verstärkung der ammoniakalischen Enzephalopathie.

\section{Kurzkettige Fettsäuren}

Die Serum- und Liquorkonzentrationen von kurzkettigen Fettsäuren (Propionat, Butyrat, Valeriat, Octonoat) sind bei Patienten mit Coma hepaticum erhöht $(14,15)$. Nach intraperitonealer Injektion können sie ein reversibles Coma auslösen. Die kurzkettigen Fettsäuren entstehen wahrscheinlich infolge inkompletter $\beta$-Oxidation langkettiger Fettsäuren im Darm und werden nur unvollständig in der Leber katabolisiert. Ihre toxischen Wirkungen betreffen vorwiegend die Hemmung einiger Enzyme (z. B. der Glykolyse, $\mathrm{Na}^{+}-\mathrm{K}^{+}$-ATPase), die Hemmung der $\mathrm{NH}_{4}^{+}$-Entgiftung durch Harnstoffsynthese, die kompetitive Hemmung der Bindung von anderen Toxinen an Albumin und den Synergismus mit anderen zerebrotoxischen Substanzen wie Ammonium und Mercaptanen (4). Auf die letztgenannte Wirkung weist die Beobachtung hin, daß die Serumkonzentrationen kurzkettiger Fettsäuren im allgemeinen nur wenig mit dem Schweregrad der Enzephalopathie korrelieren (16) und daß die Coma-auslösende Wirkung des Ammoniaks durch gleichzeitige Gabe von kurzkettigen Fettsäuren tierexperimentell erheblich gesteigert werden kann (17).

\section{Mercaptane und andere Methioninderivate}

Im Serum sowie im Liquor und im Gehirn von Patienten mit hepatogener Enzephalopathie kommt es zu einer oft exzessiven (ca. 30fachen) Erhöhung der Methioninkonzentration bei gleichzeitigem Auftreten zerebrotoxischer Oxidationsprodukte wie Methioninsulfoxid und Methioninsulfoxim (18). Im Colon entstehen durch bakterielle Umsetzungen aus dieser Aminosäure die extrem toxischen, schwefelhaltigen Mercaptane, wie Methanthiol, Ethanthiol, Dimethylsulfid und Dimethyldisulfid, deren Konzentrationen im Blut, Urin, Liquor und Hirn wegen reduzierter hepatischer Clearance stark erhöht sind, was zur Diagnostik des Comas herangezogen werden kann (19) (s.u.). Ihre Anwesenheit in der Atemluft der Comapatienten erzeugt den typisch süßlichen Geruch des "Foetor hepaticus". Die comaauslösende Wirkung der Mercaptane ist ebenso wie ihre toxizitätssteigernde Wirkung von Ammonium und kurzkettigen Fettsäuren tierexperimentell bewiesen (5). Eine orale Methioninzufuhr bewirkt auf diesem Wege eine Verstärkung des Co- mas. Die toxische Wirkung der Mercaptane beruht dabei wahrscheinlich auf einer neuronalen Membranfunktionsstörung infolge Hemmung der $\mathrm{Na}^{+}-\mathrm{K}^{+}$- ATPase und $\mathrm{zu}$ sätzlich auf einer Hemmung der $\mathrm{NH}_{3}$-Entgiftung.

\section{Phenole und Phenolderivate}

Phenole entstehen überwiegend im Darm als Abbauprodukte der aromatischen Aminosäuren Tyrosin und Phenylalanin und werden nach Absorption in der Leber durch Veresterung mit Glukuronsäure und Schwefelsäure entgiftet. Im Coma hepaticum kommt es zu einer starken Erhöhung der freien (nicht-konjugierten) Phenole im Serum und Urin bei gleichzeitigem Auftreten von Phenolderivaten ( $\mathrm{p}$-Hydroxyphenylazetat, p-Hydroxyphenyllaktat u.a.) $(3,20,21)$. Das Ausmaß der Konzentrationserhöhung geht parallel mit dem klinischen Schweregrad der Enzephalopathie, doch ist der pathobiochemische Mechanismus ihrer zerebrotoxischen Wirkung noch nicht genau bekannt.

\section{Neurotransmitterhypothese}

Diese Hypothese geht von den Veränderungen der Neurotransmitterzusammensetzung im zentralen Nervensystem aus, die zu Hemmungen in der synaptischen Erregungsübertragung führen $(4-6,8)$. Ursache hierfür sind primär die komplexen Verschiebungen des Aminosäureprofils im Serum (22-24), die sich zeigen in einer

- Zunahme der aromatischen Aminosäuren Tyrosin und Phenylalanin,

- Zunahme des freien, d.h. nicht-proteingebundenen Anteils des Tryptophans,

- Zunahme von Methionin, Asparaginsäure und Glutaminsäure,

- Abnahme der verzweigtkettigen Aminosäuren Leucin, Isoleucin und Valin.

Das molare Verhältnis von verzweigtkettigen Aminosäuren zur Summe von Phenylalanin und Tyrosin von normalerweise $3-4$ ist auf 1 und weniger erniedrigt $(4,23)$. Der Pathomechanismus der Verschiebung des Aminosäurespektrums bei Leberzirrhose und/oder portosystemischem Umgehungskreislauf ist komplex. Die Zunahme der aromatischen Aminosäuren Phenylalanin und Tyrosin beruht auf einer reduzierten hepatischen Aufnahme der enteral freigesetzten und absorbierten Aminosäuren und auf einer Einschränkung ihres Katabolismus in der Leber.

Die Aktivität des weitgehend leberspezifischen Schlüsselenzyms des Phenylalaninabbaus, die Phenylalaninhydroxylase, welche die Degradation durch 4-Hydroxylierung zum Tyrosin einleitet, ist in der zirrhotischen Leber signifikant vermindert (25). Die Tryptophangesamtkonzentration im Serum ist nur mäßig erhöht, doch findet sich eine starke Zunahme des nicht-proteingebundenen, freien Anteils (26). Dazu trägt einerseits die häufig vorhandene Hypalbuminämie bzw. Hypoproteinämie (ein additiver Faktor des Comas) (Abb. 1), andererseits die kompetitive Verdrängung des Tryptophans von Transportproteinen durch erhöhte Konzentration freier Fettsäuren (s.o.) bei. Die Abnahme der verzweigtkettigen Aminosäuren hingegen ist keine direkte Folge einer Leberfunktionsstörung, da diese Aminosäuren vorwiegend in extrahepatischen Geweben wie Gehirn, Muskel und Niere katabolisiert werden (6). Ihre Clearancerate ist bei hepatischer Enzephalopathie erhöht $(27,28)$. Sie wird 
vermutlich durch eine erhöhte Insulinsekretion bewirkt, die die Aufnahme mit nachfolgendem oxidativem Abbau dieser Aminosäuren im Muskel stimuliert. Die Erhöhung des Insulinspiegels wiederum erfolgt reaktiv auf die durch Hyperammoniämie erzeugte, oft stark ausgeprägte $\mathrm{Hy}$ perglukagonämie (29). Folge des vermehrten oxidativen Abbaus der verzweigtkettigen Aminosäuren ist eine Nettoproduktion von Ammoniak, die zu dessen Konzentrationserhöhung beiträgt und somit im Rahmen eines Circulus vitiosus den gesteigerten Katabolismus der Aminosäuren Valin, Leucin und Isoleucin unterhält $(4,29)$. Als weiterer pathobiochemisch möglicherweise bedeutsamer Ort der Verwertung dieser Aminosäuren muß die Niere mit ihrer Fähigkeit zur Glukoneogenese angesehen werden.

Die zerebralen Auswirkungen der peripheren Aminosäureverschiebungen erklären sich daraus, daß

a) die Aminosäuren Tyrosin und Phenylalanin Substrate der intrazerebralen Synthese der adrenergischen Neurotransmitter Dopamin und Noradrenalin sind (Abb. 3),

b) die Aminosäure Tryptophan Vorstufe der intrazerebralen Bildung des inhibitorischen Neurotransmitters Serotonin ist (Abb. 3).

c) die neutralen Aminosäuren Phenylalanin, Tyrosin, Tryptophan und Methionin mit den verzweigtkettigen Aminosäuren Leucin, Valin und Isoleucin um ein gemeinsames Aminosäure-Carriersystem durch die Blut-HirnSchranke konkurrieren (30). Da mit der Abnahme der verzweigtkettigen Aminosäuren ein wichtiger Kompetitor um dieses Transportsystem wegfällt, kommt es zu erhöhtem Influx der neutralen Aminosäuren in den Liquorraum, was überdies durch ihre stark erhöhte Serumkonzentration noch verstärkt wird. Hier besteht eine für die Therapie der hepatischen Enzephalopathie nutzbare Korrekturmöglichkeit durch Infusion verzweigtkettiger Aminosäuren, die zu einer Hemmung des Hirneinstroms aromatischer Aminosäuren führt (31).

Die erhöhte intrazerebrale Phenylalaninkonzentration bewirkt eine kompetitive Hemmung der Tyrosin 3-Monooxygenase, das Schrittmacherenzym in der Bildung der physiologischen Neurotransmitter Dopamin und Noradrenalin, da Tyrosin und Phenylalanin über ähnliche Affinitäten zu diesem Enzym verfügen. Tryptophan führt ebenfalls zu einer Hemmung dieses Enzyms. Damit wird nicht nur die Bildung von Dopa und somit von Dopamin und Noradrenalin inhibiert, sondern gleichzeitig durch Blockierung des Tyrosinabbaus und Steigerung der Tyrosinbildung aus Phenylalanin dessen Konzentration weiter erhöht. Das im Exzess vorhandene Tyrosin wird in einem alternativen Stoffwechselweg zunächst decarboxyliert zu Tyramin, welches durch $\beta$-Hydroxylierung mittels einer relativ unspezifischen Dopamin- $\beta$-Monooxygenase in Octopamin überführt wird (Abb. 3). Octopamin wirkt als falscher (inaktiver) Neurotransmitter (32). Dieses Amin wird in Kompetition mit Dopamin und Noradrenalin in die zentralen Nervenendigungen aufgenommeh, gespeichert

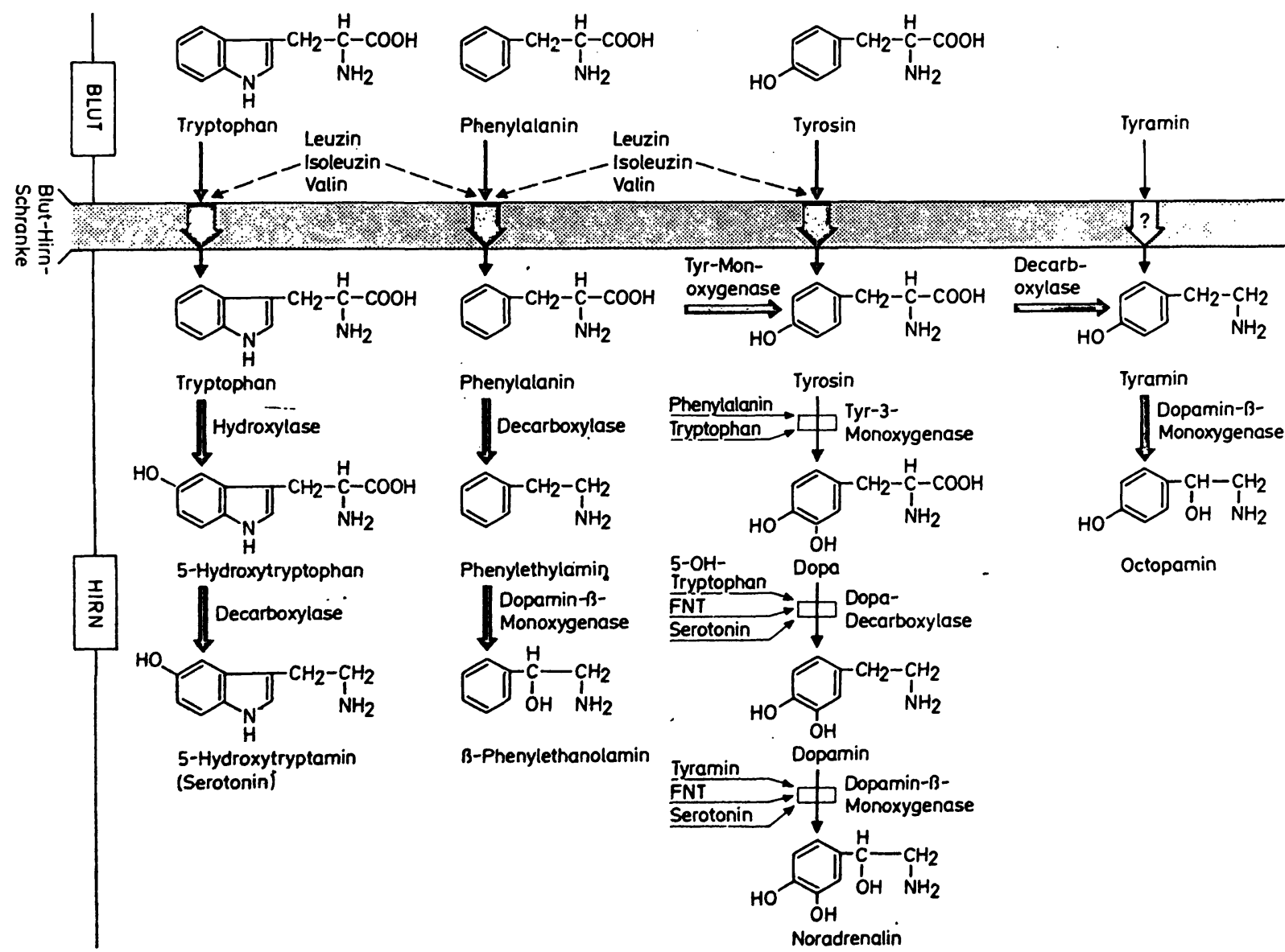

Abb. 3: Veränderungen der intracerebralen Neurotransmittersynthese bei hepatischer Enzephalopathie und Coma hepaticum. Die Synthesewege falscher Neurotransmitter (FNT) und inhibitorischer Transmitter (Serotonin) sind angegeben. $\square$ symbolisiert die Hemmung einer enzymatischen Umwandlung, $\Rightarrow$ symbolisiert die Verstärkung eines Stoffwechselweges. Erklärung im Text 
und durch Depolymerisationsreize freigesetzt, entfaltet jedoch postsynaptisch nur etwa 1/so der sympathomimetischen Wirkung von Noradrenalin (6). Die Synthesehemmung der echten Neurotransmitter und ihre kompetitive Verdrängung von den synaptischen Nervenendigungen durch den schwachen Neurotransmitter Octopamin führen zu einer hochgradigen Beeinträchtigung der synaptischen Erregungsübertragung. Ein weiterer falscher Neurotransmitter mit ähnlicher Struktur und Wirkung ist $\beta$-Phenylethanolamin, welches, ausgehend von dem erhöhten Phenylalaninangebot, vermehrt gebildet wird (33). Zusätzlich zu ihren inhibitorischen Wirkungen auf die Synapsenfunktion hemmen die falschen Neurotransmitter auf mehreren Ebenen die Synthese von Dopamin und Noradrenalin, so daß sich auch hier ein pathobiochemischer Circulus vitiosus ausbilden kann (6). Beide falschen Neurotransmitteramine sind bei Comapatienten in erhöhter Konzentration im Serum und Urin nachweisbar $(32,34)$. Da das Ausmaß ihrer Erhöhung mit dem Schweregrad des Comas gut korreliert, ist ihre Bestimmung zur Diagnostik und Verlaufskontrolle des Coma hepaticum empfohlen worden (Tab. 1) (s.u.) $(32,34,35)$.

Tab. 1: Klinisch-chemische Parameter zur Diagnose und Verlaufskontrolle der hepatischen Enzephalopathie und des Coma hepaticum

\begin{tabular}{|c|c|c|c|}
\hline $\begin{array}{l}\text { Parameter } \\
\text { im Serum }\end{array}$ & $\begin{array}{l}\text { Pathomechanismus des } \\
\text { Konzentrationsanstieges }\end{array}$ & Interpretation & Literatur \\
\hline \multirow[t]{2}{*}{ Ammoniak } & \multirow{2}{*}{$\begin{array}{l}\text { reduzierte hepatische } \mathrm{NH}_{3} \text {-Elimina- } \\
\text { tion } \\
\text { - verminderte Harnstoffsynthese } \\
\text { - portosystemische Umgehung } \\
\text { erhöhte enterale } \mathrm{NH}_{3} \text {-Bildung } \\
\text { - gastrointestinale Blutungen } \\
\text { - Exzess-Proteinzufuhr u.a. }\end{array}$} & $\begin{array}{l}\text { Ausmaß der Hyperammoniämie kor- } \\
\text { reliert nicht streng mit Schwere- } \\
\text { grad der Enzephalopathie ( } 10 \% \text { der } \\
\text { Comapatienten haben normale } \\
\mathrm{NH}_{3}-\text { Konzentrationen im arteriellen } \\
\text { Blut) }\end{array}$ & \multirow[t]{2}{*}{$\begin{array}{l}5,6,7,9,13,40 \\
43,48\end{array}$} \\
\hline & & $\begin{array}{l}\text { Verstärkende Faktoren der } \mathrm{NH}_{3}- \\
\text { Toxizität und } \mathrm{NH}_{3}-\text { Erhöhung } \\
\text { - Alkalose (z. B. Diuretika) } \\
\text { - Hypokaliämie (z. B. Diuretika, se- } \\
\text { kundärer Hyperaldosteronismus) } \\
\text { - katabole Zustände (z. B. Infektio- } \\
\text { nen) }\end{array}$ & \\
\hline
\end{tabular}

Octopamin gesteigerte intrazerebrale Synthese aus Tyrosin bzw. Tyramin
Ausmaß der Konzentrationserhöhung im Serum (und Urin) korreliert eng mit dem Schweregrad der Enzephalopathie

Geeignet zur Diagnose und Überwachung der hepatischen Enzephalopathie

Mäßige Octopaminanstiege auch bei Urämie

Phenylethanolamin gesteigerte intrazerebrale Synthese aus Phenylalanin wie bei Octopamin, gute Korrelation mit dem Schweregrad der Enzephalopathie
$32,35,44,45$

\section{4}

starke Erhöhung bei akutem Leberversagen (Leberzerfallscoma)

normale oder leicht erhöhte Konzentrationen bei Leberausfallscoma

zur Differenzierung beider Comaformen geeignet

-mäßige Erhöhung bei Zirrhotiker ohne Enzephalopathie

19,47 reduzierte hepatische Clearance de
intestinal gebildeten Mercaptane starke Erhöhung bei Zirrhotiker mit Enzephalopathie

Korrelation mit dem Schweregrad der Enzephalopathie

häufig bei Leberzerfallscoma erhöht,

19 seltener bei Leberausfallscoma

Anstieg nur bei schweren Formen des Comas, prognostisch ungünstig $_{e}$

mäßige (ca. 2x) Erhöhung bei Zirrhotikern ohne Enzephalo36

reduzierter hepatischer Abbau erhöhte extrahepatische Bildung aus Tyrosin pathie

starke (ca. 5x) Erhöhung bei Zirrhotikern mit Enzephalopathie 
Die Zunahme der Tryptophankonzentration im Liquor und Gehirn ist Ausgangspunkt einer vermehrten Synthese von Serotonin (5-Hydroxytryptamin), einem inhibitorisch wirkenden Neurotransmitteramin. Die Konzentrationen des Serotonins und seines Metaboliten 5-Hydroxyindolazetat sind stark erhöht im Liquor von Comapatienten (6). Außer seiner hemmenden Wirkung auf die synaptische Erregungsübertragung greift es zusammen mit seinen Vorstufen hemmend in die Synthese der echten Neurotransmitter ein (Abb. 3). So inhibiert Tryptophan die Aktivität der Tyrosin 3-Monooxygenase, 5-Hydroxytryptophan und -tryptamin hemmen kompetitiv die Dopa-Decarboxylase und Serotonin die Dopamin- $\beta$ Monooxygenase (Abb. 3). Daraus resultiert wiederum eine Hemmung der Noradrenalinsynthese auf mehreren Ebenen.

Die bei leberzirrhotischen Patienten mit Enzephalopathie nachgewiesene Erhöhung der Serum-Tyraminkonzentration wird ebenfalls als ein möglicher pathogenetischer Faktor des Coma hepaticum betrachtet (36). Tyramin entsteht im Intestinaltrakt durch bakterielle Decarboxylierung des Tyrosins und wird physiologischerweise in der Leber durch Monoaminooxydasen vollständig metabolisiert. Die eingeschränkte Clearance-Funktion der zirrhotischen Leber, die portosystemische Shuntbildung, aber auch eine gesteigerte extrahepatische Umwandlung des erhöht vorliegenden Tyrosins in Tyramin sind die wahrscheinlichen Ursachen der Hypertyraminämie (36). Da eine intakte Blut-Hirn-Schranke für phenolische Amine weitgehend undurchlässig ist, dürfte sich die Tyraminämie nur bei erhöhter Permeabilität, wie sie im fortgeschrittenen Stadium des Comas nachweisbar ist, pathogenetisch auswirken. Die zerebralen Wirkungen des Tyramins liegen auf verschiedenen Ebenen und betreffen vorwiegend seine indirekte sympathomimetische Wirkung (Freisetzung von Noradrenalin), die Hemmung der Noradrenalinsynthese und durch $\beta$-Hydroxylierung die vermehrte Bildung des falschen Neurotransmitters Octopamin (Abb. 3), dessen inhibitorische Wirkung bereits erwähnt wurde.

Eine weitere, tierexperimentell begründete Neurotransmitterhypothese weist dem $\gamma$-Aminobutyrat (GABA) als inhibitorischen Transmitter eine Rolle in der Pathogenese der Enzephalopathie bzw. des Comas zu $(37,38)$. Diese Annahme basiert auf dem Nachweis erhöhter intrazerebraler GABA-Konzentrationen und einer durch GABAinduzierten Zunahme postsynaptischer spezifischer Rezeptoren. Die Veränderungen führen zu einer vermehrten Sensitivität des Hirns gegenüber GABAergischer neuronaler Hemmung. GABA entsteht extrazerebral vorwiegend im Colon und kann unter Umgehung der insuffizienten Leber in die systemische Zirkulation und über eine abnorm permeable Blut-Hirn-Schranke in das Hirn gelangen (37). Ob diesem GABA-Mechanismus auch im Coma hepaticum des Menschen eine entscheidende Funktion zukommt, kann derzeit noch nicht definitiv entschieden werden (8). Auf die mögliche Rolle des Glutamins als falscher oder inaktiver Transmitter ist bereits bei Beschreibung der toxischen Wirkung des Ammoniaks hingewiesen worden (s.o.).

Zusammenfassend sind die Verschiebungen der neuronalen Transmitterzusammensetzung im Coma hepaticum somit charakterisiert durch eine Abnahme der exzitatorischen Neurotransmitter Dopamin und Noradrenalin, aber auch von Glutamat und Aspartat und durch eine Zunahme der inaktiven (falschen) Neurotransmitter Octopamin und seiner Vorstufen Tyramin, $\beta$-Phenylethanolamin und Glutamin sowie durch erhöhte Konzentratio- nen inhibitorischer Substanzen wie Serotonin und möglicherweise GABA.

\section{Klinisch-chemische Diagnostik und Verlaufskontrolle der hepatogenen Enzephalopathie}

Die Bestimmung einiger der im pathobiochemischen Teil erwähnten Metabolite im Serum oder Urin, seltener im Liquor, kann mit Einschränkungen zur Diagnostik, Verlaufskontrolle, Abschätzung des Schweregrades, zur Unterscheidung der beiden Comaformen (Zerfalls- und Ausfallscoma) sowie zur prognostischen Beurteilung der hepatischen Enzephalopathie und des Coma hepaticum herangezogen werden und somit die klinische und elektroenzephalographische Bewertung der Enzephalopathie unterstützen. Für die genannten Fragestellungen eignen sich bevorzugt die folgenden Parameter (siehe auch Tab. 1):

\section{Diagnostik}

Ammonium im arteriellen (weniger zuverlässig im venösen) Blut

Obwohl die Bestimmung der Ammoniumkonzentration die am häufigsten durchgeführte klinisch-chemische Untersuchung bei hepatischer Enzephalopathie ist, sollte bei der Interpretation der Hyperammoniämie einschränkend berücksichtigt werden, daß

a) nur etwa $90 \%$ der Comapatienten erhöhte Konzentrationen aufweisen (verläßlicher wäre eine Ammoniumbestimmung im Liquor) $(11,39)$ und somit eine fehlende Hyperammoniämie nicht gegen die Diagnose eines Coma hepaticums sprechen muß.

b) das Ausmaß der Hyperammoniämie nicht streng den Schweregrad des Comas wiedergibt, da, wie oben ausgeführt wurde, eine Vielzahl synergistischer Pathomechanismen wirksam sind.

c) Extrahepatische Mechanismen (Abb. 2) die Ammoniumkonzentration im Serum maßgeblich mitbestimmen.

d) Die Toxizität des Ammoniums nicht nur von seiner Geamtkonzentration, sondern wesentlich von der $\mathrm{pH}$-abhängigen Lage des Dissoziationsgleichgewichtes $\mathrm{NH}_{4}^{+} \rightleftharpoons$ $\mathrm{NH}_{3}+\mathrm{H}^{+}$abhängig ist. Mit zunehmendem $\mathrm{pH}$ (metabolische und respiratorische Alkalose) entsteht das frei diffusible, neuro-toxische $\mathrm{NH}_{3}$.

e) Coma-unabhängige genetische und erworbene Hyperammoniämiesyndrome existieren (40).

Ein wesentlicher Vorteil des Ämmoniums gegenüber allen anderen unten aufgeführten biochemischen Parametern der Enzephalopathie kommt von der Seite des Labors. Er ist gegeben in der vergleichsweise guten Praktikabilität der enzymatisch-UV-optischen Bestimmungsmethode $(41,42)$ bei Berücksichtigung wichtiger Störfaktoren (43).

\section{Octopamin, $\beta$-Phenylethanolamin, Mercaptane} und Tyramin im Serum und Urin (Tab. 1)

Ihre Konzentrationsbestimmung ist aufwendiger (Gaschromatographie, HPLC, enzymatisch-radiochemisch etc.) als die des Ammoniums (enzymatisch-optisch) und deshalb im Rahmen der Diagnostik im allgemeinen spezielleren Fragestellung vorbehalten. 


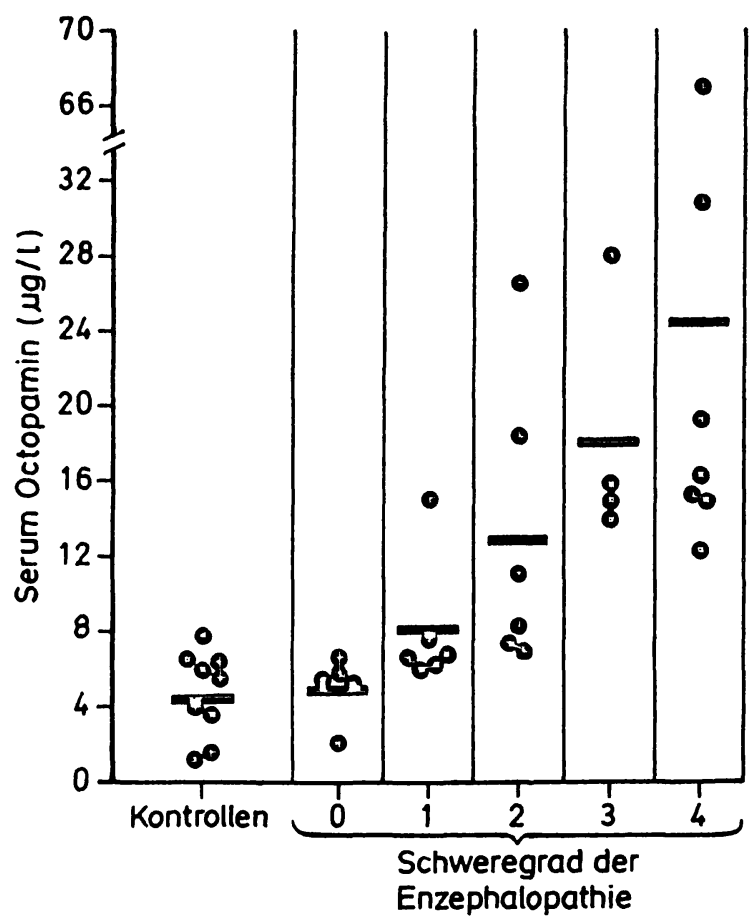

Abb. 4: Octopaminkonzentrationen im Serum von Patienten mit verschiedenen neuro-psychiatrischen Schweregraden der portosystemischen (hepatogenen) Enzephalopathie (nach Ref. 44)

\section{Verlaufskontrolle und Abschätzung des Schweregrades}

Octopamin, $\beta$-Phenylethanolamin, Methanthiol (Methylmercaptan) und weniger zuverlässig Tyramin und freie Phenole im Blut und Urin (Tab. 1)

Octopamin im Serum ist ein sehr sensitiver Parameter der hepatischen Enzephalopathie $(32,44,45)$, das Ausmaß der Konzentrationserhöhung korreliert streng mit dem neuro-psychiatrischen Schweregrad des Comas $(32,44)$ (Abb. 4). Der Parameter ist deshalb gut zur Verlaufskontrolle geeignet. Im Vergleich zur Bestimmung des Octopamins im Serum ist der Nachweis seiner erhöhten Ausscheidung im Urin klinisch weniger zuverlässig $(32,44)$. Bei primären Nierenerkrankungen ohne Leberbeteiligung kann es ebenfalls zu mäßiger Erhöhung der Serumkonzentration und Urinausscheidung des Octopamins kommen (32). Nachteilig für den Routineeinsatz dieses Parameters ist die gegenwärtig noch aufwendige Bestimmungsmethode, der ein radio-enzymatisches Verfahren mit Phenylethanolamin-N-Methyltransferase zugrunde liegt (46). Phenylethanolamin ist sowohl in seiner klinischen Bedeutung und vom analytischen Aufwand her dem Octopamin vergleichbar (34), die übrigen Parameter, wie z.B. Methanthiol (47), sind für eine Verlaufskontrolle weniger bedeutungsvoll als Octopamin.

\section{Unterscheidung von Leberzerfalls- und -ausfallscoma}

Ethanthiol im Serum (Tab. 1)

Obwohl die Differenzierung beider Comaformen meistens mit klinischen Mitteln möglich ist, weisen stark erhöhte (ca. 10fach) Ethanthiolkonzentrationen im Serum auf ein Leberzerfallscoma hin. Leberausfallscoma geht mit normalen oder subnormalen Ethanthiolkonzentrationen einher. Geringe Erhöhungen (ca. 2fach) finden sich auch bei chronisch aktiver Hepatitis und Verschlußikterus (19). Die Bestimmung der Marcaptane (Ethanthiol, Methanthiol, Dimethylsulfid) erfolgt mit der Gaschromatographie und ist unter dieser apparativen Voraussetzung für ein Routinelabor geeignet $(19,47)$.

\section{Prognostik}

Dimethylsulfid im Serum (Tab. 1)

Dieses Mercaptan ist im Serum Gesunder nicht nachweisbar, sondern tritt nur bei sehr schweren, prognostisch ungünstigen Comaverläufen (vorwiegend Leberzerfallscoma) auf (19). Sein Anstieg im Serum weist frühzeitig auf eine fatale Entwicklung der Enzephalopathie hin.

Die aufgeführten Parameter können auch im Liquor in erhöhten Konzentrationen nachgewiesen werden. In dieser Körperflüssigkeit ist jedoch die Glutaminkonzentration der zuverlässigste Parameter für den Schweregrad der hepatischen Enzephalopathie und allen anderen Kenngrößen im Serum überlegen.

Schrifttum:

1. TREY, C., DAVIDSON, C. S.: The Management of Fulminant Hepatic Failure. Progr. Liver Disease (POPPER, H., SCHAFFNER, F., Hrsg.). Grune \& Stratton, New York 1970, Bd. III, 282.

2. KALK, H., WILDHIRT, E.: Krankheiten der Leber. In: Klinik der Gegenwart. Urban \& Schwarzenberg 1958, Bd. 7, 377.

3. MÜTING. D., REIKOWSKI, J.: Coma hepaticum. Grundlagen der Pathogenese und der Behandlung. Fortschr. Med. 101, 1610-1614 (1983).

4. ZIEVE, L.: Hepatic Encephalopathy: Summary of Present Knowledge with an Elaboration on Recent Developments. Progr. Liver Disease (POPPER, $H$. SCHAFFNER, F., Hrsg.). Grune \& Stratton, New York 1979, Bd. VI, 327-341. 5. ZIEVE, L.: The Mechanism of Hepatic Coma. Hepatology 1, 360-365 (1981).

6. FISHER, J. E., BALDESSARINI, R. J.: Pathogenesis and Therapy of Hepatic Coma.

Progr. Liver Disease (POPPER, H., SCHAFFNER, F., Hrsg.). Grune \& Stratton, New York 1976, Bd. V. 363-397.

7. HOYUMPA, A. M., DESMOND, P. V., AVANT, G. R., ROBERTS, R. K., SCHENKER S.: Hepatic Encephalopathy. Gastroenterology 76, 184-195 (1979).

8. HOYUMPA, A. M., SCHENKER, S.: Perspectives in Hepatic Encephalopathy. J. Lab. Clin. Med. 100, 477-487 (1982).

9. MAIER, K. P., TALKE, H., GEROK, W.: Activities of Urea-Cycle Enzymes in Chronic Liver Disease. Klin. Wschr. 57, 661-665 (1979).

10. SMITH, H. H., Jr, SMITH, L. S.: WAIS Finctioning of Cirrhotic and Non-Cirrhotic Alcoholics. J. Clin. Psychol. 33, 309-313 (1977).

11. SUMMERSKILL, W. H. J., WOLFE, S. J.: The Metabolism of Ammonia and $\alpha$ Keto Acids in Liver Disease and Hepatic Coma. J Clin. Invest. 36, 361-372 (1957)

12. BALDESSARINI, R. J., YORKE, C.: Uptake and Release of Possible False Transmitter Amino Acids by Rat Brain Tissue. J. Neurochem. 23, 839-848 (1974).

13. GEROK. W.: Biochemische Aspekte der hepatischen Enzephalopathie. Internistische Welt 10, 343-347 (1980)

14. TAKAHASHI, Y.: Serum Lipids in Liver Disease. Liver Disease and the Relationship of Serum Lipids and Hepatic Coma. Jpn. J. Gastroenterol. 60, 571-579 (1963).

15. CHEN, S., MAHADEVAN, V., ZIEVE, L.: Volatile Fatty Acids in the Breath of Patients with Cirrhosis of the Liver. J. Lab. Clin. Med. 75, 622-627 (1970).

16. WALKER, C. O., MCCANDLESS, D. W., MCGARRY, J. D., SCHENKER, S. Cerebral Energy Metabolism in Short Chain Fatty Acid-Induced Coma. J. Lab. Clin. Med. 76, 569-583 (1970).

17. ZIEVE, F. J., ZIEVE, L., DOIZAKI, W. M., GILSDORF, R. B.: Synergism between Ammonia and Fatty Acids in the Production of Coma: Implications for Hepatic Coma. J. Pharmacol. Exp. Ther. 191, 1.0-16 (1974)

18. GEROK, W.: Aminosäurenstoffwechsel bei Leberinsuffizienz. Verh. Dtsch. Ges. Inn. Med. 75, 33-43 (1969).

19. BRUNNER, G. SCHARFF, P.: Untesuchungen über den diagnostischen Wert der Bestimmung von Mercaptanen im Serum bei Lebererkrankungen. Disch. med. Wschr. 103, $1796-1800$ (1978).

20. MÜTING, D., KRAUS, W., STUMPF, U., DEESZ, H., KELLER, H. E.: Die klinische Bedeutung der freien Phenole bei Leberkrankheiten. Med. Welt 22, 368-372 (1971). 21. RUGE, W., OTTO, P.: Der Stoffwechsel phenolischer Verbindungen bei Lebererkrankungen mit encephalopathischen Erscheinungen. Med. Welt (NF) 149-153 (1972).

22. FISCHER, J. E.: Amino Acids in Hepatic Coma. Digest. Dis. Sci. 27, 97-102 (1982).

23. McCULlOUgh, J. A., CZAJA, A. J., JONES, J. D., GO, V. L. W.: The Nature and Prognostić Significance of Serial Amino Acid Determinations in Severe Chronic Active Liver Disease. Gastroenterology 81, 645-652 (1981).

24. ZOLI, M., MARCHESINI, G., ANGIOLINI, A., DONDI, C., BIANCHI, B., PISI, E.: Plasma Amino Acids as Markers of Liver Dysfunction in Cirrhotics. Scand. J. Gastroent. 16, 689-692 (1981).

25. LEVINE, R. J., CONN, H. O.: Tyrosine Metabolism in Patients with Liver Disease. 25. LEVINE, R. J., CONN, H. O.: Tyrosine
J. Clin. Invest. 46, 2012-2020 (1967).

26. FENSTROM, J. D., WURTMAN, R. J.: Brain Serotonin Content: Physiological Regulation by Plasma Neutral Amino Acids. Science 178, 414-416 (1972). 
27. IOB, V., MATTSON, W. J., Jr, SLOAN, M., COON, W. W., TURCOTTE, J. G. CHILD, C. G.: Alterations in Plasma-Free Amino Acids in Dogs with Hepatic Insufficiency. Surg. Gynecol. Obstet. 130, 794-801 (1970).

28. MCMENAMY, R. J., VANG, J. H., DRAPANAS, T.: Amino Acids and $\alpha$-Keto Acid Concentrations in Plasma and Blood of the Liverless Dog. Am. J. Physiol. 209, 10461052 (1965).

29. JAMES, J. H., ZIPARO, V., JEPPSON, B., FISCHER, J. E.: Hyperammonemia, Plasma Amino Acid Imbalance and Blood Brain Amino Acid Transport: A Unified Theory of Portal-Systemic Encephalopathy. Lancet 2, 772-775 (1979).

30. ORLOWSKI, M. G., SESSA, J. P., GREEN, J. P.: $\gamma$-Glutamyl Transpeptidase in Brain Capillaries: Possible Site of a Blood Brain Barrier for Amino Acids. Science 184 66-68 (1974).

31. WAHREN, J. DENIS, J. DESURMONT, P., ERIKSSON, L. S., ESCOFFIER, J. M., GAUTHIER, A. P. HAGENFELDT, L., MICHEL, H., OPOLON, P., PARIS, J.-C. VEYRAC, M.: Is Intravenous Administration of Branched Chain Amino Acids Effective in the Treatment of Hepatic Encephalopathy? A Multicenter Study. Hepatology 3. 475-480 (1983).

32. LAM, K. C., TALL, A. R., GOLDSTEIN, G. B., MISTILIS, S. P.: Role of a False Neurotransmitter, Octopamine, in the Pathogenesis of Hepatic and Renal Encephalopathy. Scand. J. Gastroent. 8, 465-472 (1973).

33. KOPIN. I. J.: False Adrenergic Transmitters. Ann. Rev. Pharm. 8, 377-394 (1968).

34. CANGIANO, C., ROSSI-FANELLI, A., BOZZI, V. CALCATERRA, A., CASCINO A.. CAPOCACCIA, L.: Plasma Phenylethanolamine in Hepatic Encephalopathy. Europ. Journ. of Clin. Invest. 8, 183-184 (1978).

35. ROSSI-FANELLI, F., CANGIANO, C., ATTILI, A., ANGELICO, M., CASCINO, A. CAPOCACCIA, L., STROM, R., CRIFO, C.: Octopamine Plasma Levels and Hepatic Encephalopathy: A Re-Appraisal of the Problem. Clin. Chim. Acta 67, 255-261 (1976).

36. FARAJ, B. A., BOWEN, P. A., JAMES, J. W., RUDMAN, D.: Hypertyraminemia in Cirrhotic Patients. Now Engl. Journ. of Med. 294, 1360-1364 (1976).

37. SCHAFER, D. F., JONES, E. A.: Hepatic Encephalopathy and the $\gamma$-AminobutyricAcid Neurotransmitter System. Lancet 1, 18-20 (1982).

38. ROBERTS, E.: The $\gamma$-Aminobutyric Acid (GABA) System and Hepatic Encephalopathy. Hepatology 4, 342-345 (1984).

39. PHEAR, E. A., SHERLOCK, S., SUMMERSKILL, W. H. J.: Blood-Ammonium Levels in Liver Disease and "Hepatic Coma". Lancet 1, 836-840 (1955).

40. FLANNERY, D. B., HSIA, Y. E., WOLF, B.: Current Status of Hyperammonemic Syndromes. Hepatology 2, 495-506 (1982)
41. DA FONSECA-WOLLHEIM, F.: Bedeutung von Wasserstoffionenkonzentration und ADP-Zusatz bei der Ammoniakbestimmung mit Glutamatdehydrogenase. Z. Klin. Chem. Klin. Biochem. 11, 421-425 (1973).

42. DA FONSECA-WOLLHEIM, F.: Direkte Plasmaammoniakbestimmung ohne Enteiweißung. Z. Klin. Chem. Klin. Biochem. 11, 426-431 (1973).

43. PRELLWITZ, W., KAPP, S., DENNEBAUM. R.: Methodische Untersuchungen und klinische Bedeutung des Blutammoniaks. Med. Welt 27, 1277-1280 (1976).

44. MANGHANI, K. K., LUNZER, M. R., BILLING, B. H., SHERLOCK, S.: Urinary and Serum Octopamine in Patients with Portal-Systemic Encephalopathy. Lancet 1, 943946 (1975).

45. CAPOCACCIA, L., CANGIANO, C., ATTILI, A. F., ANGELICO, M., CASCINO, A. ROSSI-FANELLI, F.; Octopamine and Ammonia Plasma Levels in Hepatic Encephalopathy. Clin. Chim. Acta 75, 99-105 (1977).

46. MOLINOTT, P. B., LANDSBERG, L., AXELROD, J.: An Enzymatic Assay for Octopamine and other Beta-Hydroxylated Phenylethylamines. J. Pharmacol. Exp. Ther. 170, 253-261 (1969).

47. MCCLAIN, C. J.. ZIEVE, L., DOIZAKI, W. M., GILBERSTADT, S., ONSTAD, G. R. Blood Methanethiol in Alcoholic Liver Disease with and without Hepatic Encephalopathy. Gut 21, 318-321 (1980).

48. LOCKWOOD, A. H., MCDONALD. J. M., REIMAN, R. E., GELBARD, A. S. LAUGHLIN, J. S., DUFFY, T. E., PLUM, F.: The Dynamics of Ammonia Metabolism in Man. J. Clin. Invest. 63, 449-460 (1979).

Anschrift des Verfassers:

Prof. Dr. A. M. Gressner

Abteilung für Klinische Chemie und Zentrallaboratorium Phillips-Universität Marburg

Baldingerstraße

3550 Marburg/Lahn 